Ольга Кислищььна

\title{
НАЦИОНАЛЬНЫЙ ИНДЕКС \\ КАЧЕСТВА ЖИЗНИ (БЛАГОПОЛУЧИЯ) \\ КАК ИНСТРУМЕНТ МОНИТОРИНГА ЭФФЕКТИВНОСТИ СОЦИАЛЬНО-ЭКОНОМИЧЕСКОЙ ПОЛИТИКИ В РОССИИ
}

\begin{abstract}
В статье представлена оценка динамики качества жизни россиян за период с 2000 по 2014 гг. Методология основана на построении методом процентных отношений композитного индекса - интегрального показателя, агрегирующего десять основных аспектов качества жизни (здоровье и здравоохранение, образование и обучение, доходы, жилье, работа, безопасность, семья, культура и отдых, управление, состояние окружающей среды), измеряемых посредством 82 индикаторов, выделенных на основе литературных обобщений. В качестве информационной базы использованы не только объективные статистические данные Росстата, «Трансформационного Мониторинга в целях более эффективного обеспечения социальной справедливости» (TransMonEE), Европейской базы данных «Здоровье для всех» (HFA-DB), но и субъективные: данные специальных обследований (Российский мониторинг экономики и здоровья ВШЭ (РМЭЗ), опросы Левада-Центра. Установлено, что в целом качество жизни в России с 2000 г. увеличилось на $26.2 \%$. Выявлены аспекты и индикаторы, повлиявшие на этот процесс, и, напротив, снижающие качество жизни. Из десяти аспектов, формирующих благополучие, улучшение произошло по восьми. Основной вклад в процесс улучшения качества жизни внесли такие аспекты как семья (+70.9\%), доходы (+60.6\%), безопасность (+55.4\%). Хуже всего обстоят дела в сфере окружающей среды (-19.5\%). Из 82 индикаторов, составляющих аспекты качества жизни, улучшился 51, а 31, соответственно, ухудшился. Больше всего негативных изменений произошло в следующих сферах: состояние окружающей среды (5 из 7 индикаторов ухудшились), образование и обучение (8 из 12), культура и отдых (3 из 6), работа (4 из 10). Полученные
\end{abstract}

Ольга Анатольевна Кислицына - д.э.н., зав. сектором проблем измерения качества жизни, Институт экономики РАН, Москва, Россия. Электронная почта: olga.kislitsyna@gmail.com 
результаты могут быть использованы для мониторинга эффективности социально-экономической политики в России и выработки мер, направленных на повышение качества жизни россиян.

Ключевые слова: качество жизни, благополучие, интегральный показатель, индекс качества жизни, индекс благополучия

DOI: $10.17323 / 727-0634-2017-15-4-547-558$

\section{Введение}

В течение долгого периода времени ВВП служил единственным индикатором социально-экономического развития общества, хотя экономистразработчик, Саймон Кузнец, был против его использования таким образом (цит. по Wilson, Tyedmers 2013). Все чаще исследователи говорят об ограничениях ВВП (напр., Hicks 1946; Samuelson 1961; Mishan 1967; Nordhaus, Tobin 1972; Hirsch 1976; Sen 1976; Hartwick 1990; Tinbergen, Hueting 1992; Dasgupta, Mäler 2000; Kahneman et al. 2004; Van den Bergh 2009) и приходят к выводу, что «настало время перенести акцент с измерения экономического производства на измерение благополучия людей» (Stiglitz et al. 2009). При этом наравне с термином «благополучие» используются такие понятия как «качество жизни», «счастье», «удовлетворенность жизнью», «процветание» (Кислицына 2016а). Инициативы по измерению социально-экономического прогресса и благополучия начаты во многих странах (Кислицына 2016b).

Большинство исследователей и организаций, занимающихся проблемами качества жизни (благополучия), твердо уверены, что оно должно быть основным приоритетом лиц, ответственных за разработку социальноэкономической политики. Проведенные исследования показали, что преимущества обеспечения высокого уровня благополучия заключаются, по крайней мере, в следующем (Diener, Seligman 2004): (1) оно способствует хорошему здоровью (самооценке здоровья), увеличивает продолжительность жизни, вероятность благоприятного для здоровья поведения, снижает частоту психопатологий, в то время как низкий уровень качества жизни создает угрозу иммунной системе и повышает риск определенных заболеваний; (2) высокий уровень качества жизни тесно связан с высоким уровнем брачности и стабильностью браков, ростом числа друзей и социальной поддержки; (3) работники с высоким качеством жизни более организованы, трудятся с высокой производительностью и прибыльностью.

На основе проведенного анализа литературы была разработана и апробирована методология измерения благополучия (качества жизни) в России. Целью исследования было измерить и проследить динамику качества жизни россиян. Это позволяет предоставить лицам, ответственным за принятие решений, полезную информацию по качеству жизни в России, а также привлечь внимание общественности к этой проблеме. 


\section{Данные и методы}

В качестве информационной базы для исследования использованы не только объективные статистические данные Росстата, Трансформационного Мониторинга в целях более эффективного обеспечения социальной справедливости (TransMonEE) и Европейской базы данных «Здоровье для всех» (HFA-DB) за период 2000-2014 гг., но и субъективные данные специальных обследований: Российский мониторинг экономики и здоровья ВШЭ (РМЭ3) и опросы Левада-Центра. Для измерения качества жизни (благополучия) построен композитный индекс - интегральный показатель, который объединяет отдельные аспекты благополучия.

Необходимо отметить отсутствие среди исследователей единого мнения о том, как должен выглядеть композитный индекс не только с точки зрения основных аспектов и измеряющих их индикаторов, но и методологии построения, т.е. того, что касается нормализации, взвешивания, агрегации аспектов и индикаторов (Кислицына 2016c). При построении композитного индекса качества жизни возникают три главные проблемы. Первые две касаются выбора аспектов благополучия и измеряющих их индикаторов. Есть два подхода к этому процессу: выбор осуществляется на основе опроса широкого круга лиц, являющихся специалистами в различных областях; или на основе теоретических представлений об объективных факторах, которые необходимы людям для достижения хорошего качества жизни. Первая проблема заключается в субъективности этого выбора, независимо от подхода. Исследователи отмечают (Gasper 2010), что выбор объективных индикаторов благополучия в какой-то степени отражает ценности составителей списка, а также ценности общества. Для построения композитного индекса качества жизни в России основные аспекты и характеризующие их индикаторы отобраны на основе анализа (1) отчетов международных органов и организаций, зарубежных и отечественных исследовательских групп, отдельных исследователей ${ }^{1}$, некоторые из которых разработали подходы для регулярного измерения благополучия; (2) научной и методической литературы, публикаций, дающих представление об объективных факторах, которые необходимы людям для того, чтобы иметь хорошее качество жизни ${ }^{2}$.

Вторая проблема связана с недоступностью в отечественной статистике многих индикаторов, которые часто используются для построения национальных индексов качества жизни в других странах. Особенно это касается таких аспектов как, например, управление (вопросы государственного администрирования), общество (социальные отношения и социальная сплоченность). По мнению исследователей, индикаторы, в первую очередь, должны обладать такими характеристиками, как доступность для анализа в течение

\footnotetext{
${ }^{1}$ См. электронное Приложение 1 по ссылке: https://jsps.hse.ru/article/view/7301/8178

${ }^{2}$ См. электронное Приложение 2 по ссылке: https://jsps.hse.ru/article/view/7301/8179
} 
длительного периода времени, чувствительность к изменениям (Trewin, Hall 2010), сосредоточенность на результатах - другими словами, они должны измерять не то, сколько, например, потрачено на оказание медицинской помощи, а то, насколько успешна борьба с болезнями или насколько удовлетворены пациенты самой медицинской помощью (Kroll 2011). Между исследователями также существует консенсус, что помимо объективных показателей благополучия необходимо рассматривать субъективные: не ограничиваться только мониторингом условий, в которых живут люди, но и выяснить, насколько они довольны этими условиями (Stiglitz et al. 2009). Поэтому в дополнение к данным официальных источников во многих странах на регулярной основе проводятся обследования качества жизни населения. Этот опыт мог бы оказаться полезным и в нашей стране.

Третья проблема при построении композитного индекса состоит в выборе весовых значений для различных аспектов индекса. В настоящее время наиболее точным и популярным (Decancq, Lugo 2013) является присвоение аспектам и измеряющим их индикаторам одинаковых весов, что и было сделано при построении индекса качества жизни (благополучия) в России.

Методология построения индекса основана на использовании метода процентных отношений по аналогии с канадским индексом благополучия (Canadian Index of Wellbeing 2011). Для каждого аспекта вычислено среднее арифметическое процентных изменений индикаторов (2000 г.- базовый год), составляющих аспект; общий индекс представляет собой невзвешенное среднее арифметическое всех аспектов. На рисунках значения аспектов и индикаторов в базовый год (2000 г.) принимаются за ноль.

Индикаторы, используемые для построения индекса, имеют как отрицательный смысл (т.е. увеличение численного значения индикатора указывает на сокращение благополучия), так и положительный (т. е. увеличение численного значения индикатора указывает на увеличение благополучия). Положительный или отрицательный смысл индикаторов оцениваются, исходя из общечеловеческих ценностей (например, рождаемость имеет положительный смысл, а смертность - отрицательный). Процентные изменения для индикаторов с положительным смыслом рассчитываются по формуле $\mathrm{I}_{\mathrm{t}} / \mathrm{I}_{\mathrm{b}}$ *100, для индикаторов с отрицательным смыслом - по формуле $\left(1 / \mathrm{I}_{\mathrm{t}}\right) /\left(1 / \mathrm{I}_{\mathrm{b}}\right) * 100$, где $\mathrm{I}_{\mathrm{t}}-$ значение индикатора в год $\mathrm{t}, \mathrm{I}_{\mathrm{b}}$ - значение индикатора в год, который принят за базовый (2000 г.).

На рисунках (см. электронное приложение 3) для индикаторов с положительным смыслом график, демонстрирующий процентные изменения, имеет восходящий тренд, если наблюдается увеличение численного значения индикатора (например, увеличивается рождаемость), и нисходящий тренд в противном случае. Для индикаторов с негативным смыслом график процентных изменений имеет восходящий тренд, если наблюдается снижение численного значения индикатора (например, снижается заболеваемость туберкулезом), и нисходящий тренд в противном случае. 


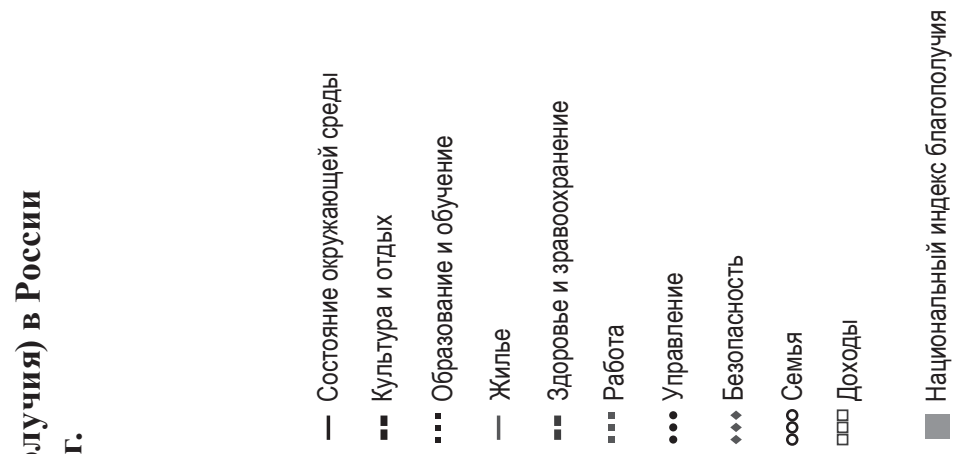




\section{Результаты.}

\section{Период 2000-2014 гr.}

На рисунке 1 представлена динамика индекса качества жизни (благополучия) в России и составляющих его аспектов за период с 2000 г. по 2014 г. В целом качество жизни увеличилось на $26.2 \%$. Из десяти аспектов, формирующих благополучие, улучшение произошло по восьми. Основной вклад в процесс улучшения качества жизни внесли такие аспекты как семья $(+70.9 \%)$, доходы (+60.6\%), безопасность $(+55.4 \%)$. Хуже всего обстоят дела в сфере окружающей среды $(-19.5 \%)$.

Из 82 индикаторов, составляющих аспекты качества жизни (см. электронное приложение 2), улучшились 51, а 31, соответственно, ухудшились. Больше всего негативных изменений произошло в следующих сферах: состояние окружающей среды (пять из семи индикаторов ухудшились), образование и обучение (восемь из 12), культура и отдых (три из шести), работа (четыре из десяти). Остановимся подробнее на аспектах, составляющих индекс, и на индикаторах, их измеряющих.

Здоровье и здравоохранение. Если разложить результирующий показатель на составляющие, то улучшения в сфере здоровья за период с 2000 г. по 2014 г. связаны с резким сокращением материнской и детской (до 5 лет) смертности, а также смертности трудоспособного населения, от инфекционных и паразитарных болезней, сердечно-сосудистых заболеваний, с уменьшением заболеваемости туберкулезом, ростом числа россиян, удовлетворенных своим здоровьем (См. электронное приложение 3 , Рис. 1) 1 . Эти тенденции перевесили негативное влияние роста заболеваемости ВИЧ, числа больных психическими расстройствами и расстройствами поведения, которым оказывается консультативно-лечебная помощь. Наблюдаются неблагоприятные тенденции, связанные с политикой «оптимизации» медицинских учреждений - беспрецедентным сокращением не только числа больничных коек, но и врачебных амбулаторно-поликлинических учреждений. Увеличивается доля расходов домохозяйств на услуги здравоохранения. Можно ожидать, что подобная «оптимизация» в скором времени приведет к негативным социально-демографическим последствиям.

Образование и обучение. Несмотря на рост числа выпускников вузов и увеличение доли занятых в экономике с высшим образованием, в сфере образования и обучения наблюдаются негативные тенденции: в высшем образовании - растет доля обучающихся заочно, с полным возмещением средств на обучение, доля выпущенных специалистов социально-гуманитарного, управленческого и экономического профиля; в среднем общем образовании - сокращается число общеобразовательных организаций

\footnotetext{
${ }^{1}$ См. электронное Приложение 3 по ссылке: https://jsps.hse.ru/article/view/7301/8180
} 
и увеличивается число учеников, приходящихся на одного учителя (См. электронное приложение 3, Рис. 2). Эти тенденции в перспективе неблагоприятно отразятся на качестве образования, что можно видеть на примере с каждым годом снижающихся результатов участия России в международных математических олимпиадах. Несмотря на рост охвата детей соответствующего возраста дошкольным образованием, остается проблема с обеспеченностью местами в дошкольных учреждениях.

Работа. Основные тенденции, которые обеспечили улучшение в этой сфере за период 2000-2014 гг.- рост средней заработной платы, сокращение безработицы, численности работающих более 40 часов в неделю (См. электронное приложение 3 , Рис. 3). Положительное влияние этих факторов перевесило негативный вклад увеличения доли занятых в неформальном секторе экономики, занятых во вредных и (или) опасных условиях труда и сокращение численности фактически действующих ИП.

Доходы. Рост индекса доходов обусловлен увеличением доходов, сокращением численности населения с доходами ниже прожиточного минимума, снижением доли расходов на питание в общих расходах домохозяйств, повышением уверенности населения в будущем (ростом доли тех, кто думает, что его семья через 12 месяцев будет жить лучше), снижением доли тех, кто, по их мнению, стоит на первых трех ступенях лестницы благосостояния, т.е. самых бедных (См. электронное приложение 3 , Рис. 4). На высоком уровне остается неравенство в распределении доходов.

Жилье. Небольшое улучшение в сфере жилья связано с увеличением площади жилых помещений, приходящихся на одного человека, улучшением качества жилья (доли жилищ, оснащенных канализацией, отоплением, горячим водоснабжением, водопроводом), повышением уровня доступности жилья (снижением стоимости квадратного метра), ростом числа семей, улучшивших жилищные условия (в \% от нуждающихся, стоящих на учете) в результате действия программы «Доступное жилье» (См. электронное приложение 3, Рис. 5). Отрицательный вклад вносит рост доли ветхого и аварийного жилья.

Безопасность. Существенное повышение безопасности обусловлено снижением как общего уровня преступности, так и отдельных ее видов, таких как убийства, изнасилования и преступления против собственности (кражи), сокращением смертности от ДТП (См. электронное приложение 3, Рис. 6). В то же время намечается рост преступлений против детей и преступности, связанной с незаконным оборотом наркотиков.

Семья. Значительный вклад в улучшение качества жизни вносят положительные изменения в сфере семьи, что, в первую очередь, объясняется снижением числа абортов (См. электронное приложение 3 , Рис. 7). Одновременно выросла рождаемость, увеличилось число браков, снизилось количество детей, рожденных вне брака. Однако количество разводов продолжает расти. 
Культура и отдых. Оценить в цифрах культурный уровень людей оказалось труднее всего. Некоторые косвенные показатели свидетельствуют о неблагоприятных тенденциях, которые наблюдаются в сфере культуры и отдыха, что объясняется снижением количества парков культуры и отдыха, учреждений культурно-досугового типа, охвата населения библиотечным обслуживанием (См. электронное приложение 3, Рис. 8).

Управление. Улучшение ситуации в сфере управления с 2000 г. связано с ростом числа людей, позитивно оценивающих положение дел в стране и одобряющих действия правительства (См. электронное приложение 3, Рис. 9). Также снизилась доля тех, кто считает себя бесправным. Аспект «Управление» мог бы демонстрировать большие успехи, если бы не продолжающийся рост преступлений, квалифицированных как взяточничество.

Состояние окружающей среды. Наибольший негативный вклад в качество жизни в России вносит состояние окружающей среды, обусловленный ежегодным увеличением лесной площади, пройденной пожарами и одновременно сокращением площади лесовосстановления, ростом отходов производства и потребления и снижением доли обезвреженных и использованных отходов (См. электронное приложение 3 , Рис. 10). Более благополучно обстоит дело с состоянием водных ресурсов и охраной атмосферного воздуха.

\section{Период 2012-2014 rr.}

Отдельно рассмотрим период с 2012 г., с которого в стране начался полномасштабный экономический кризис, сопровождаемый ухудшением социально-экономического положения населения. Качество жизни продолжило свой рост (+3.5\%), но темп этого роста замедлился. Из десяти аспектов благополучия улучшение произошло по шести. В основном рост качества жизни за этот период обусловлен улучшением в сфере управления $(+24 \%)$, безопасности $(+12.3 \%)$, семьи (+7\%). Первая тенденция связана с резким увеличением доли одобряющих деятельность правительства и позитивно оценивающих положение дел в стране, что, вероятно, объясняется «крымскими» событиями 2014 г. Вторая тенденция определяется улучшением в сфере безопасности - снижением уровня смертности от убийств и ДТП, уменьшением преступности против личности и собственности, а также некоторым снижением преступлений против детей. Наконец, третья тенденция обусловлена дальнейшим снижением числа абортов.

Сдерживают рост качества жизни негативные тенденции, которые с 2012 г. наблюдаются в таких показателях, как доходы (-9.6\%), работа $(-2.6 \%)$, жилье $(-2.1 \%)$, состояние окружающей среды (-2.1\%). Негативные изменения в сфере доходов объясняются ухудшением практически всех индикаторов, составляющих этот аспект: снижаются среднедушевые денежные доходы в сравнении с прожиточным минимумом, растет доля населения с доходами 
ниже прожиточного минимума, увеличивается доля расходов домохозяйств на продукты питания и безалкогольные напитки, сокращается уверенность в завтрашнем дне и растет доля домохозяйств, ставящих себя на первые три ступени «лестницы благосостояния» (самые бедные). Основной вклад в ухудшение аспекта «Работа» вносят сокращение среднемесячной заработной платы в сравнении с прожиточным минимумом трудоспособного, существенный рост доли работающих 40 часов в неделю и больше, продолжающиеся тенденции сокращения числа фактически действующих ИП, увеличение доли занятых в неформальном секторе экономики, доли занятых во вредных и (или) опасных условиях труда. Ухудшение в области жилья связано, в основном, с продолжающимся с 2010 г. сокращением доли семей, улучшивших свои жилищные условия, а также с некоторым снижением доли качественного жилья, благоустроенного водопроводом, канализацией, отоплением, горячим водоснабжением.

Состояние окружающей среды, которое с 2000 г. вносит наибольший негативный вклад в динамику качества жизни в России, продолжило ухудшаться за период с 2012 г. по 2014 г., что связано с продолжающимися негативными тенденциями: ростом площади лесных пожаров и одновременным снижением лесовосстановления, увеличением выбросов загрязняющих веществ в атмосферный воздух от передвижных источников, ростом отходов и снижением доли переработанных и использованных отходов.

\section{Выводы}

Таким образом, в результате литературных обобщений выделены основные аспекты и измеряющие их индикаторы для оценки качества жизни населения в России; собраны статистические данные с 2000 по 2014 гг; рассчитаны интегральные показатели качества жизни и его аспектов при условии равенства весов внутри групп и между группами аспектов и индикаторов; проведен анализ показателей, что позволило сделать вывод об улучшении качества жизни с 2000 г. Выявлены основные аспекты и измеряющие их индикаторы, оказывающие позитивное и негативное влияние на качество жизни россиян.

Проведенное исследование имеет несколько ограничений, связанных с выбором индикаторов. Во-первых, оно, возможно, использует не все индикаторы, которые могут характеризовать качество жизни, в силу их отсутствия в отечественной статистике или короткого срока наблюдения. Во-вторых, полученные результаты во многом зависят от качества как официальных статистических данных, так и субъективных данных опросов населения. Несмотря на эти ограничения, проведенное исследование имеет важное значение, т.к. представляет собой первую пробную версию национального индекса качества жизни (благополучия) в России. Методика оценки качества жизни открыта для обсуждения и корректив. 


\section{Список источников}

Кислицына О. (2016а) Измерение качества жизни/благополучия: международный опыт, М.: Институт экономики РАН.

Кислицына О. (2016b) Новое глобальное движение: измерение прогресса и благополучия. Проблемы современной экономики, (3): 81-86.

Кислицына О. (2016с) Подходы к измерению качества жизни (благополучия). Экономический анализ: теория и практика, 10 (457):28-38.

Canadian Index of Wellbeing (2011) How are Canadians Really Doing? Highlights: Canadian Index of Wellbeing 1.0, Waterloo, ON: University of Waterloo. Доступно по ссылке: https://uwaterloo.ca/canadian-index-wellbeing/sites/ca.canadian-index-wellbeing/files/uploads/files/CIW-HowAreCanadiansReallyDoing-FINAL_0.pdf (дата обращения: 12 октября 2017).

Dasgupta P., Mäler K. G. (2000) Net National Product, Wealth, and Social Well-Being. Environment and Development Economics, 5 (1-2): 69-93.

Decancq K., Lugo M. A. (2013) Weights in Multidimensional Indices of Wellbeing: An overview. Econometric Reviews, 32 (1): 7-34.

Diener E., Seligman M. (2004) Beyond Money: Toward an Economy of Well-Being. Psychological Science in the Public Interest, 5 (1): 1-31.

Gasper D. (2010) Understanding the Diversity of Conceptions of Well-Being and Quality-oflife. Journal of SocioEconomics, (39):351-360.

Hartwick J. M. (1990) Natural Resources, National Accounting and Economic Depreciation. Journal of Public Economics, (43): 291-304.

Hicks J. R. (1946) Value and Capital. Vol. 2, Oxford: Clarendon Press.

Hirsch F. (1976) Social Limits to Growth, Cambridge, MA: Harvard University Press.

Kahneman D., Krueger A., Schkade D., Schwarz N., Stone A. (2004) Toward National WellBeing Accounts. American Economic Review, (94): 429-434.

Kroll C. (2011) Measuring Progress and Well-Being Achievements and Challenges of a New Global Movement. Berlin: Friedrich-Ebert-Stiftung. Доступно по ссылке: http://library.fes. de/pdf-files/id/ipa/08509.pdf (дата обращения: 1 июня 2017).

Mishan E. J. (1967) The Cost of Economic Growth, London: Staples Press.

Nordhaus W.D., Tobin J. (1972) Is Growth Obsolete? Economic Research: Retrospect and Prospect. (5). Доступно по ссылке: http://www.nber.org/books/nord72-1 (дата обращения: 1 июня 2017).

Samuelson P.A. (1961) The Evaluation of 'Social Income': Capital Formation and Wealth. F. A. Lutz, D.C. Hague (eds.) The Theory of Capital, London: MacMillan:32-57.

Sen A. (1976) Real National Income. Review of Economic Studies, 43 (1): 19-39.

Stiglitz J., Sen A., Fitoussi J. (eds.) (2009) Report by the Commission on the Measurement of Economic Performance and Social Progress, Paris: The Commission.

Tinbergen J., Hueting R. (1992) GNP and Market Prices: Wrong Signals for Sustainable Economic Success that Mask Environmental Destruction. R. Goodland, H. Daly, S. E. Serafy (eds.) Population, Technology and Lifestyle: The Transition to Sustainability, Washington DC: Island Press: 52-62.

Trewin D., Hall J. (2010) Developing Societal Progress Indicators: A Practical Guide, Paris: OECD.

Van den Bergh J. (2009) The GDP Paradox. Journal of Economic Psychology, (30): 117-135.

Wilson J., Tyedmers P. (2013) Rethinking What Counts. Perspectives on Wellbeing and Genuine Progress Indicator Metrics from a Canadian Viewpoint. Sustainability, (5): 187-202. 
Olga Kislitsyna

\section{THE NATIONAL INDEX FOR THE QUALITY OF LIFE (WELL-BEING) AS A TOOL FOR MONITORING THE EFFECTIVENESS OF SOCIO-ECONOMIC POLICY IN RUSSIA}

The article presents an evaluation of the dynamics of the quality of life of Russians for the period from 2000 to 2014. The methodology is based on the construction of a composite index that aggregates ten main aspects of life quality: health and healthcare, education and training, income, housing, work, security, family, culture and recreation, governance, environment. These are measured according to eighty-two indicators that were identified in literature review. Objective statistical data of Rosstat and Transformation Monitoring data for more effective social justice, the European Health for All database) and subjective data (Russian Longitudinal Monitoring Survey from the HSE and Levada Centre polls) were used. It was found that, in general, quality of life in Russia has increased by $26.2 \%$ since 2000 . Several key aspects and indicators have contributed to this process, and, on the contrary, reduced the quality of life. From the ten aspects of well-being improvement was found in eight aspects. The main contribution to the process of the improvement of quality of life was made by such aspects as Family $(+70.9 \%)$, Income $(+60.6 \%)$, Security $(+55.4 \%)$. The worst situation was in the sphere of the Environment $(-19.5 \%)$. From the eighty-two indicators that measured the quality of life aspects fifty-one have improved, and thirty have worsened. Most of the negative changes occurred in the following areas: Environment (five out of seven indicators deteriorated), Education and training (eight of twelve), Culture and recreation (three of six), Work (four out of ten). The obtained results can be used to monitor the effectiveness of socio-economic policy and development of measures aimed to improve the quality of life in Russia.

Key words: quality of life, well-being, integral indicator, index of life quality, well-being index

DOI: $10.17323 / 727-0634-2017-15-4-547-558$

\section{References}

Canadian Index of Wellbeing (2011) How are Canadians Really Doing? Highlights: Canadian Index of Wellbeing 1.0, Waterloo, ON: University of Waterloo. Available at: https://uwaterloo. ca/canadian-index-wellbeing/sites/ca.canadian-index-wellbeing/files/uploads/files/CIW-HowAreCanadiansReallyDoing-FINAL_0.pdf (accessed 12 October 2017).

Olga A. Kislitsyna - Dr. Sci. (Economic), Head of the sector of measuring quality of life problems, Economic Institute of Russian Academy of Sciences, Moscow, Russian Federation. Email: olga.kislitsyna@gmail.com 
Dasgupta P., Mäler K. G. (2000) Net National Product, Wealth, and Social Well-being. Environment and Development Economics, 5 (1-2): 69-93.

Decancq K., Lugo M. A. (2013) Weights in Multidimensional Indices of Wellbeing: An Overview. Econometric Reviews, 32 (1): 7-34.

Diener E., Seligman M. (2004) Beyond Money: Toward an Economy of Well-being. Psychological Science in the Public Interest, 5 (1): 1-31.

Gasper D. (2010) Understanding the Diversity of Conceptions of Well-being and Quality-oflife. Journal of SocioEconomics, (39): 351-360.

Hartwick J. M. (1990) Natural Resources, National Accounting and Economic Depreciation. Journal of Public Economics, (43): 291-304.

Hicks J. R. (1946) Value and Capital. Vol. 2, Oxford: Clarendon Press.

Hirsch F. (1976) Social Limits to Growth, Cambridge, MA: Harvard University Press.

Kahneman D., Krueger A., Schkade D., Schwarz N., Stone A. (2004) Toward National Wellbeing Accounts. American Economic Review, 94:429-434.

Kislitsyna O. (2016a) Izmerenie kachestva zhizni/blagopoluchija: mezhdunarodnyj opyt [Measuring the Quality of Life/Well-Being: International Experience], Moscow: Institute of Economics RAS.

Kislitsyna O. (2016b) Novoe global'noe dvizhenie: izmerenie progressa i blagopoluchiya [A new Global Movement: Measuring Progress and Prosperity]. Problemy sovremennoy ekonomiki [Issues of modern economy], (3): 81-86.

Kislitsyna O. (2016c) Podkhody k izmereniyu kachestva zhizni (blagopoluchiya) [Approaches to Measuring the Quality of Life (Well-Being)]. Ekonomicheskiy analiz: teoriya i praktika [Economic Analysis: Theory and Practice], 10 (457):28-38.

Kroll C. (2011) Measuring Progress and Well-Being Achievements and Challenges of a New Global Movement. Berlin: Friedrich-Ebert-Stiftung. Available at: http:/library.fes.de/pdf-files/id/ ipa/08509.pdf (accessed 1 June 2017).

Mishan E. J. (1967) The Cost of Economic Growth, London: Staples Press.

Nordhaus W.D., Tobin J. (1972) Is Growth Obsolete? Economic Research: Retrospect and Prospect, (5). Available at: http://www.nber.org/books/nord72-1 (accessed 1 June 2017).

Samuelson P. A. (1961) The Evaluation of 'Social Income': Capital Formation and Wealth. F. A. Lutz, D.C. Hague (eds.) The Theory of Capital, London: MacMillan:32-57.

Sen A. (1976) Real National Income. Review of Economic Studies, 43 (1): 19-39.

Stiglitz J., Sen A., Fitoussi J. (eds.) (2009) Report by the Commission on the Measurement of Economic Performance and Social Progress, Paris: The Commission.

Tinbergen J., Hueting R. (1992) GNP and Market Prices: Wrong Signals for Sustainable Economic Success that Mask Environmental Destruction. R. Goodland, H. Daly, S. E. Serafy (eds.) Population, Technology and Lifestyle: The Transition to Sustainability, Washington DC: Island Press: 52-62.

Trewin D., Hall J. (2010) Developing Societal Progress Indicators: A Practical Guide, Paris: OECD.

Van den Bergh J. (2009) The GDP Paradox. Journal of Economic Psychology, (30): 117-135.

Wilson J., Tyedmers P. (2013) Rethinking What Counts. Perspectives on Wellbeing and Genuine Progress Indicator Metrics from a Canadian Viewpoint. Sustainability, (5): 187-202. 